\title{
Piezoelectric polymer actuators in a vibration isolation application
}

\author{
G. W. Bohannan ${ }^{a}$, V. H. Schmidt ${ }^{a}$, R. J. Conant ${ }^{b}$, J. Hallenberg ${ }^{b}$, C. Nelson ${ }^{a}$, A. Childs ${ }^{b}$, \\ C. Lukes ${ }^{c}$, J. Ballensky ${ }^{c}$, J. Wehri ${ }^{c}$, B. Tikalsky ${ }^{b}$, E. McKenzie $^{b}$ \\ Dept. of ${ }^{a}$ Physics, ${ }^{b}$ Mech. Eng., ${ }^{c}$ Elec. Eng., Montana State Univ., Bozeman, MT 59717
}

\begin{abstract}
We present results from development and testing of lightweight actuators made of the piezoelectric polymer PVDF. The prototype being developed is intended for microgravity applications in space and has been tested aboard NASA's Reduced Gravity Platform. The design has been driven by the requirements for a full three-dimensional (six-degreeof-freedom) environment. Incorporation of additional electrical leads into the actuators themselves may remove the need for a separate umbilical to the suspended experiment. Linear equations describing the displacement of piezoelectric bimorphs were developed and applied to the bellows actuator including the epoxy layer. Properties for the piezoelectric layers were obtained from the literature; properties for the epoxy layer were obtained through ultrasonic testing. To assess the validity of the assumed linearity of the actuator, we conducted nonlinear finite element analysis, which indicated a high degree of linearity on contraction and up to a maximum of $5 \%$ deviation on expansion to full deflection (about $6 \mathrm{~mm}$ ). We have developed and tested a proportional-plus-derivative (PD) control system for use with the actuator in one dimension using a novel folded pendulum to simulate a zero-g environment. Passive and active characteristics are both in agreement with theoretical predictions.
\end{abstract}

Keywords: Piezoelectric polymer, actuator, vibration, isolation

\section{INTRODUCTION}

A lightweight piezoelectric polymer actuator for active vibration isolation in space application has been developed. The actuator and associated vibration control system have been tested in one dimension, with excellent results. The actuator has a low effective spring constant that provides for passive vibration damping over a large frequency range. Since the actuator is in mechanical contact with the rest of the system, we can make use of passive following at very low frequencies. Passive damping is effective at frequencies well above the mass-spring resonance. Active control is applied to both adjust the effective spring constant to tune the resonant frequency and to induce near-critical damping to eliminate the resonance overshoot.

We were chosen to fly prototype systems on NASA's Reduced Gravity Platform, a KC-135A aircraft, during March 1998, 1999, and 2000. While the first two of these flight opportunities were not completely successful, we have found them to be invaluable as part of the development and testing cycle. Consequently, we have adjusted our development effort for the March 2000 flights and kept the design focused on the requirements for our ultimate goal of a full three-dimension, six-degree-of-freedom, vibration isolation system. This work represents a continuation of that reported in Refs. 1 and 2.

The use of actuators based on piezoelectric film has been proposed before, e.g. see Ref. 3. What is new is that the design has been driven specifically to allow extension to the three-dimensional environment. To this end, we have included umbilical lines that are not part of earlier designs. Additionally, we designed and tested a simpler feedback control system.

We discuss the actuator design and static testing results in Sect. 2, and dynamic testing in Sect. 3. In Sect. 4, we discuss the one-dimensional feedback control system developed for control of the actuator. In Sect. 5 , we discuss progress on design of the three-dimensional system. The final section summarizes the results and presents comments and conclusions about the development effort.

Further author information: Send correspondence to V. Hugo Schmidt, e-mail: schmidt@physics.montana.edu 


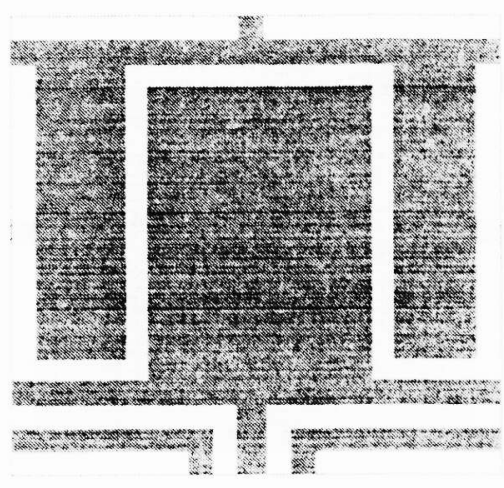

(a)

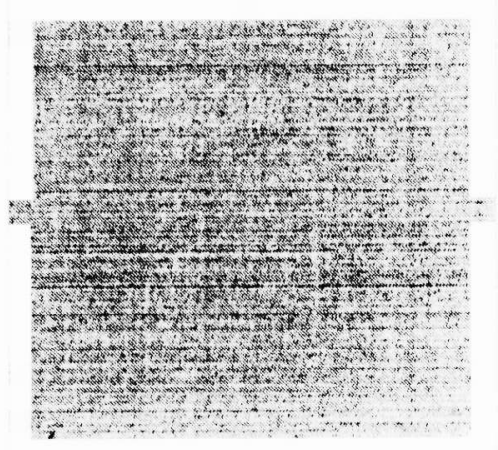

(h)

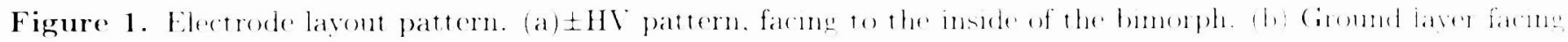
outside. Xote the two umbilical lines at bottom left and right of side (a).

Table 1. Non-zero PVDF properties. Inits of piezoelectric stran coefficients are $p(\%)$, these of the compliamere coefficients are 10$)^{10} \mathrm{~m}^{2} / \mathrm{X}$.

\begin{tabular}{|c|c|c|c|c|c|c|}
\hline Ples & $d_{31}=21$ & $d_{32}=2.3$ & $d_{33}=-26$ & $d_{24}=-27$ & $d_{1:}=$ & \\
\hline & $s_{11}=3.6 .5$ & $s_{22}=1.21$ & $s_{33}=1.72$ & $81:-1.11$ & -1.92 & \\
\hline
\end{tabular}

\section{ACTUATOR DESIGN, CONSTRUCTION, ANI STATIC TESTING}

The Space Shutfle and the International Space Station have a variety of users and systems mane of which preduce vibrat ions that are transmit ted through the rest of the plat form. Since there is very little constant bias forece le deal with. a vere low spring constant actuator can be used. This allows vibration isolation down to fractions of a deene

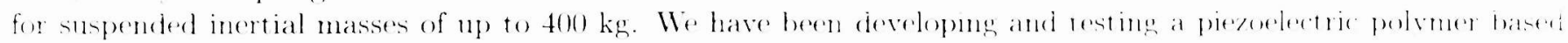
actuator for this application.

\subsection{Material properties and electrode layout}

The polymer used is a $30 \mu \mathrm{m}$ film of poly (vinylidine fluoride). (PVDF), with silver edectede larers of appoximatedy 7.5 $\mu m$ on each side. The pre-poled and electroded material has been oblained from Veasurement Specialtios. Ine. (formerly known as A.MP). The electrodes are applied with a silk screenimg process that teaters the electrode lavere highly flexible. The actuator is assembled from $5 \mathrm{~cm} \times 5 \mathrm{~cm}$ sequare sheerts with the inside and wuterde electrode patterns as shown in Fig. 1.

\subsection{PVDF and epoxy properties}

The constitutive equation for piezoelectric materials is":

$$
\{\mathbf{S}\}=[\mathbf{s}]\{\mathbf{T}\}+[\mathbf{d}]^{\dagger}\{\mathbf{E}\}
$$

where $\{\mathbf{S}\}$ is the strain matrix. $\{\mathbf{T}\}$ is the stress matrix. $\{\mathbf{E}\}$ is the electrie field matrix. $s\}$ is the compliance matrix at constant electrie field, and $[\mathbf{d}]$ is the piezoelectric strain matrix at constant stress. findicates tramsposition

Properties of the electroded PVDF were obtained from Ref. 5. which smmmarizes property data obtained by sereral researchers. Since none of the researchers provided a complete sed of propertics. We used the most complete set of properties and supplemented these with properties giren be other researchers. The values used are given in Table 1.

The epoxy used to attach the electroded PVDF sheets was 30-minute Z-poxy brand epoxy. Its properties were

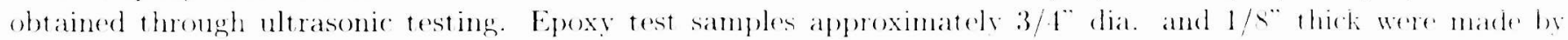
pouring epoxy into an aluminum mold. In order to mininize the number of bubbles in the epoxy. the samples were 


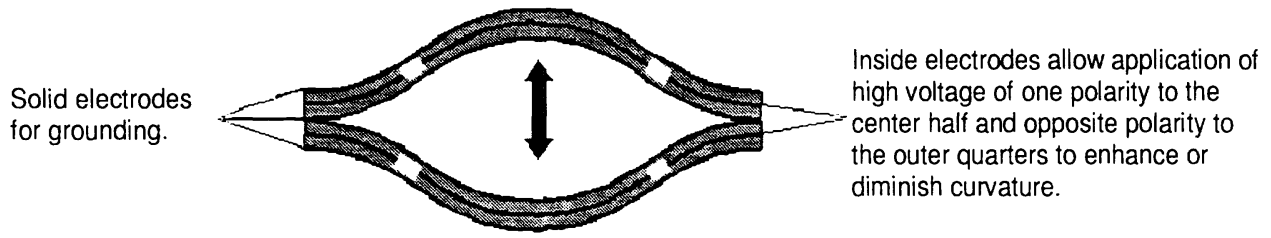

Figure 2. Bellows actuator, edge view. Expansion/contraction direction is shown by the double-ended arrow.

cured in a partial vacuum. Once cured, the sample surfaces were machined flat and then removed from the mold. The vacuum curing drew most of the bubbles to the surface where they were removed by the machining process.

The ultrasonic testing was done using 1/2" dia., $2.25 \mathrm{MHz}$ wide-band longitudinal and shear transducers. These were used to obtain the longitudinal and shear wave velocities in the material, from which were obtained the Young's modulus and Poisson's ratio. Because of the signal attenuation caused by the viscoelastic nature of the epoxy, the single transducer pulse-echo method did not produce two successive echoes when performing shear wave measurements. Therefore it was necessary to use through-transmission, which required two transducers, for these measurements. The resulting values of Young's modulus and Poisson's ratio, respectively are ${ }^{6} Y_{g}=4.2$ GPa and $\nu=0.38$.

\subsection{Bellows actuator assembly}

Electrode connector tabs of copper tape are attached to two patterned sheets. Then the two sheets are bonded together with epoxy such that the high voltage electrodes, shown in Fig. 1(a), are between the pair and the ground electrodes are on the outside, minimizing exposure of the high voltage. Epoxy coated sheets are placed in a curved form for curing to establish the pre-curved bimorph shape. The design radius of curvature is $31.5 \mathrm{~mm}$.

Epoxy is then applied to the edges of two pre-curved bimorphs and these are placed in a second form to obtain the bellows leaf spring arrangement shown in Fig. 2. Minimization and uniformity of the epoxy layer thickness are essential to obtaining a reproducible strong response. Current manufacturing techniques produce an epoxy layer thickness of $\sim 25 \mu \mathrm{m}$, but our goal is $\sim 10 \mu \mathrm{m}$.

\subsection{Bellows actuator performance prediction}

In Ref. 6, the equations governing the behavior of curved piezoelectric bimorphs in the form of circular arcs are developed and subsequently solved for the bellows configuration shown in Fig. 2. From these it can be shown that the displacement per volt is maximized when the radii of curvature of the bimorph segments are equal. The displacement, $\Delta_{V}$, at the mid-point due to an applied voltage is then given by:

$$
\Delta_{V}=8 R^{2} \frac{A}{I_{e q}} \frac{h_{e q}}{h_{f}} V d_{31}\left(\cos \phi_{0}+\phi_{0} \sin \phi_{0}-1\right) \simeq L^{2} \frac{A}{I_{e q}} \frac{h_{e q}}{h_{f}} V d_{31}
$$

in which the latter expression is the small pre-curvature limit, and where

$$
I=\frac{b h^{3}}{12}, \quad I_{e q}=2\left(I+h_{e q}^{2} A\right)+\frac{Y_{g}}{Y} I_{g}, \quad h_{e q}=\frac{h+h_{g}}{2}, \quad A=b h, \quad I_{g}=\frac{b h_{g}^{3}}{12}, \quad \phi_{0}=\frac{L}{2 R} .
$$

$\mathrm{R}$ is the mean radius of curvature of the actuator segments in Fig. $2, h_{f}, h$ and $h_{g}$ are the thickness of the bare piezoelectric film, the electroded film, and epoxy layer, respectively, $Y$ and $Y_{g}$ are the elastic modulii of the electroded piezoelectric film and epoxy layer, $b$ is the width of the actuator and $d_{31}$ is the piezoelectric strain coefficient. Additionally, $\mathrm{L}$ is the arc length from the end of the actuator to the mid-point, (i.e. one-half the length of the electroded piezoelectric film used to construct the actuator), and V is the voltage applied relative to ground.

When a force, $F$, is applied at the mid-point, the resulting deflection, $\Delta_{F}$, at the mid-point is

$$
\begin{gathered}
\Delta_{F}=\left[\frac{3\left(\phi_{0}+\cos \phi_{0} \sin \phi_{0}\right)-2\left(2 \sin \phi_{0}+\phi_{0} \cos ^{2} \phi_{0}\right)}{Y I_{e q}} R^{3}+\frac{\cos \phi_{0}+\sin \phi_{0} \cos \phi_{0}-1-\phi_{0}}{Y A_{e q}} R\right] F \\
\Rightarrow \text { (in the small curvature limit) } \frac{L^{3} F}{12 Y I_{e q}}
\end{gathered}
$$




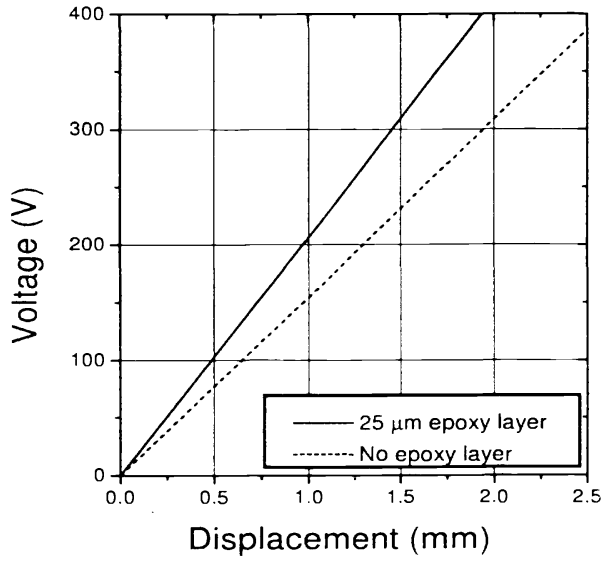

(a)

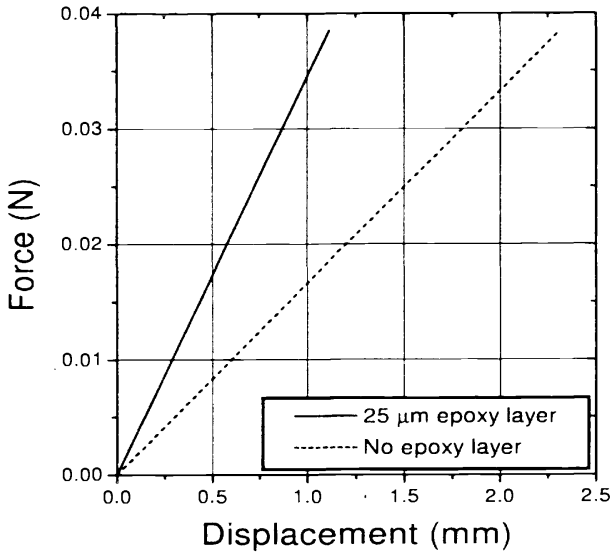

(b)

Figure 3. Predicted values of (a) voltage vs. displacement and (b) force vs. displacement for a single bellows.

where

$$
A_{e q}=2 A+\frac{Y_{g}}{Y} A_{g}, \quad \text { and } \quad A_{g}=b h_{g} .
$$

Plots of voltage versus displacement and force versus displacement are shown in Fig. 3 for the parameter values:

$$
2 L=b=4.8 \mathrm{~cm}, R=31.5 \mathrm{~mm}, h_{f}=30 \mu \mathrm{m}, h=45 \mu \mathrm{m}, h_{g}=25 \mu \mathrm{m}, Y=6.15 \mathrm{GPa}, Y_{g}=4.20 \mathrm{GPa} \text {. }
$$

Also shown are the predictions for the limit of no epoxy layer, $h_{g} \rightarrow 0$. Note that greater displacement can be achieved by minimizing the epoxy layer. In general, reducing the epoxy thickness should improve performance.

Note that $Y$ is the element $c_{11}$ of the stiffness matrix and is given in terms of the compliances $a^{7}$ :

$$
c_{11}=\frac{s_{22} s_{33}-s_{23}^{2}}{s}
$$

where

$$
s=s_{11} s_{22} s_{33}-s_{11} s_{23}^{2}-s_{22} s_{13}^{2}-s_{33} s_{12}^{2}+2 s_{12} s_{23} s_{13} .
$$

The electrical and mechanical stiffness are given by, respectively,

$$
k_{V}=V / \Delta_{V} \quad \text { and } \quad k_{F}=F / \Delta_{F} .
$$

These are plotted as a function of radius in Fig. 4. These figures show that the stiffness is quite sensitive to the radius for small values of the radius, but as the radius increases the stiffness tends to limit, which can be shown to be the stiffness for a straight actuator. The predicted mechanical and electrical stiffness for a single bellows actuator with a $25 \mu \mathrm{m}$ epoxy layer are $35 \mathrm{~N} / \mathrm{m}$ and $206 \mathrm{~V} / \mathrm{mm}$, respectively.

In order to establish the range over which the response of the actuator to an applied force was linear, the results obtained from Eq. 3 were compared with a non-linear finite element model using ANSYS. The algorithm used by ANSYS is to apply the load incrementally and update the geometry at the end of each load step to incorporate the deflection that occurs during that step. The results corroborate the prediction that on compression, the effective radius increases and the results are highly linear. On bellows extension, the radius decreases and there is a maximum of $5 \%$ deviation at full deflection for a double-bellows actuator stack (about $6 \mathrm{~mm}$ ). Since we operate the actuator within $\pm 5 \mathrm{~mm}$, this deviation is negligible and we may assume linearity over the entire operating range.

\subsection{Connectors}

In order to accommodate shear effects in the three-dimensional system, we stack two bellows assemblies at right angles to each other and attach the whole stack to the frame and box with flexible plastic line acting as a hinge. Standoff posts have been added to extend the length of the stack in order to reduce the angles resulting from shear strain. Clamps attaching the stack to the frame and box are made from aluminum blocks. Not shown in Fig. 5 are the additional electrical lines and connector for actuator control voltages and data signal lines to the umbilical. 


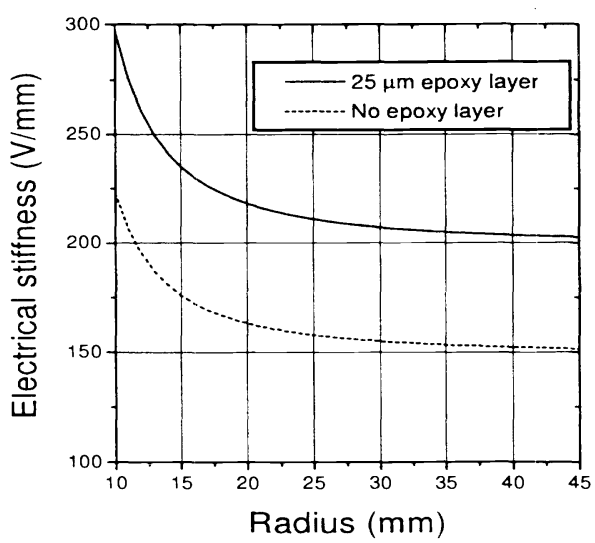

(a)

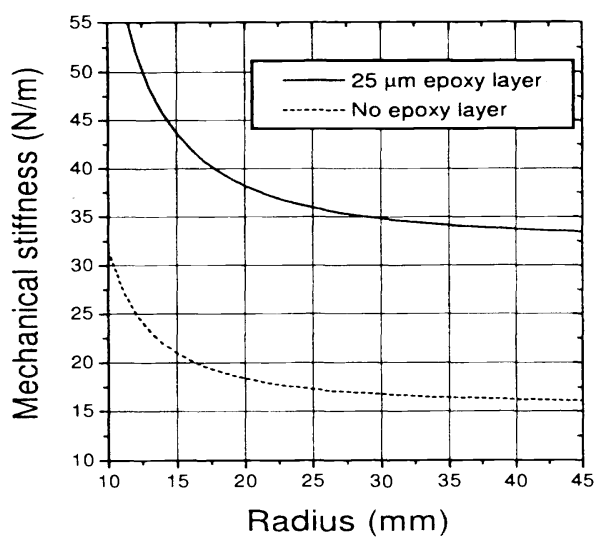

(b)

Figure 4. Predicted values of (a) electrical and (b) mechanical stiffness vs. radius for single bellows.

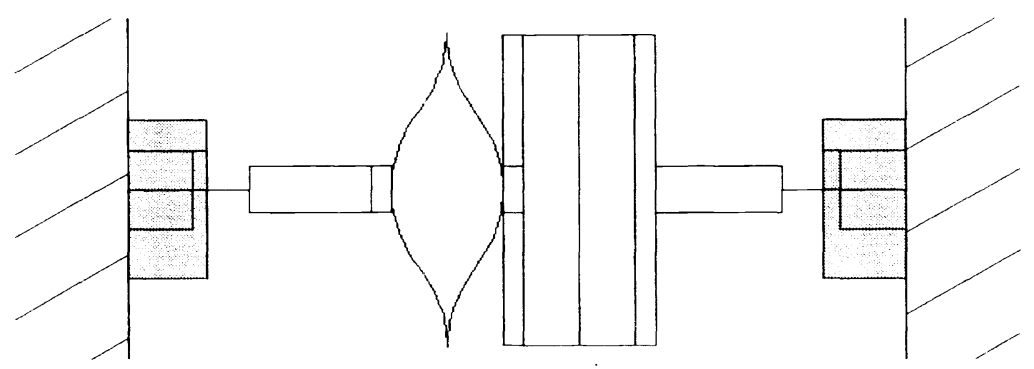

Figure 5. Completed actuator stack with two bellows structures and extension arms and hinges. One bellows structure is seen from the hollow edge and the other at $90^{\circ}$.

\subsection{Embedded umbilical lines}

Tests have shown that there is no measurable displacement effect with up to $\pm 15 \mathrm{~V}$ signals applied to the two umbilical lines. For a discussion of the umbilical problem, see, e.g., Ref. 8 .

\subsection{Prototype actuator stack characteristics}

Measured characteristics for a set of prototype double-bellows actuator stacks are summarized in Table 2. Fig. 6 displays expansion and contraction response for one of the completed double-bellows actuator stacks. As a result of the analysis above, we have been focusing our manufacturing improvement efforts on minimizing the epoxy layer. With these improvements, we anticipate cutting the electrical stiffness by nearly a factor of two. Even so, we have noted the high degree of linearity predicted by the analytic and ANSYS results.

Table 2. Prototype Actuator Stack Characteristics

\begin{tabular}{|l|l|}
\hline Parameter & Value \\
\hline \hline Spring Constant $(\mathrm{k})$ & $16.4( \pm 1.6) \mathrm{N} / \mathrm{m}$ \\
\hline Damping constant $(\mathrm{b})$ & $1.3( \pm 0.2) \mathrm{kg} \mathrm{sec}^{-1}$ \\
\hline Piezoelectric constant $(\mathrm{d})$ & $4920( \pm 194) \mu \mathrm{C} / \mathrm{N}$ \\
\hline Electrical Stiffness $\left(\mathrm{d}^{-1}\right)$ & $204( \pm 8) \mathrm{V} / \mathrm{mm}$ \\
\hline
\end{tabular}




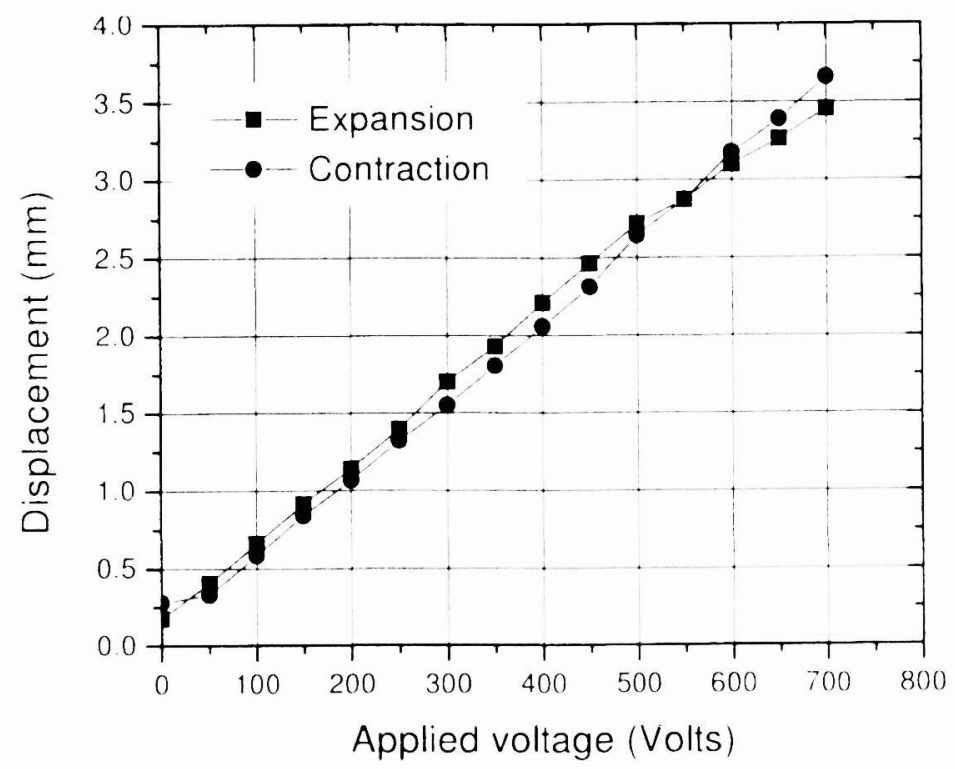

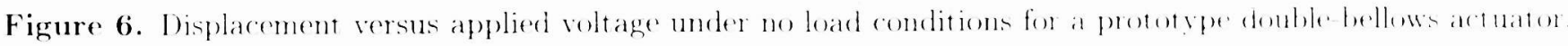

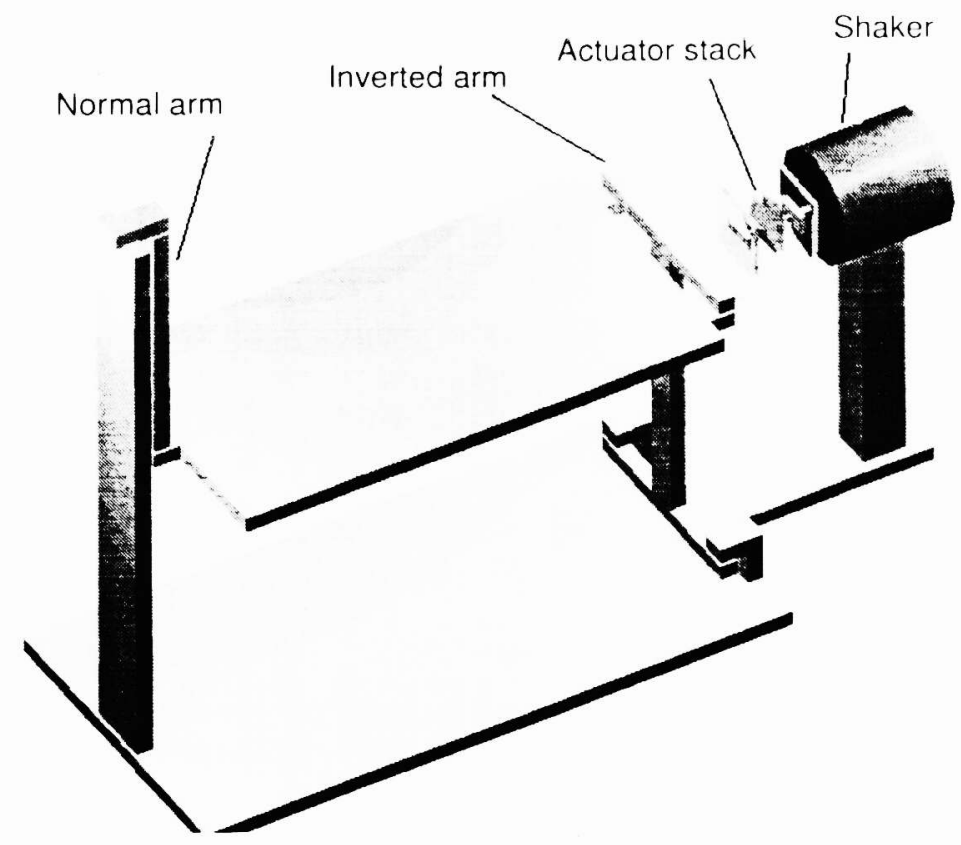

Figure 7. Folded pendulum for one-dimensional testing

\section{DYNAMIC TESTING}

For druamic testing of the double-bellows actuator stack in one dimension in the laboratory. we adapted the folded pendulum described in Refs. 9 and 10 and shown in Fig. 7. The suspended mass of the pendulum is $13.6 \mathrm{~kg}$. With careful adjustment, we achieved a free oscillation period of over 20 seconds. corresponding to less 1 han 10 pe/mom restoring force. The shaker provides excitation simulating the effect of the platform frame. Shaker freculuency and amplitude are controlled by a PC. Relative motion between the shaker head and the pendulum plat form is monitored by the same type of two-dimensional position sensing devices (PSD) we are using in the there dimensional system

Fig. 8 shows the frequency response for one of the prototype double-bellows actuator stacks across the mass-s!ning 


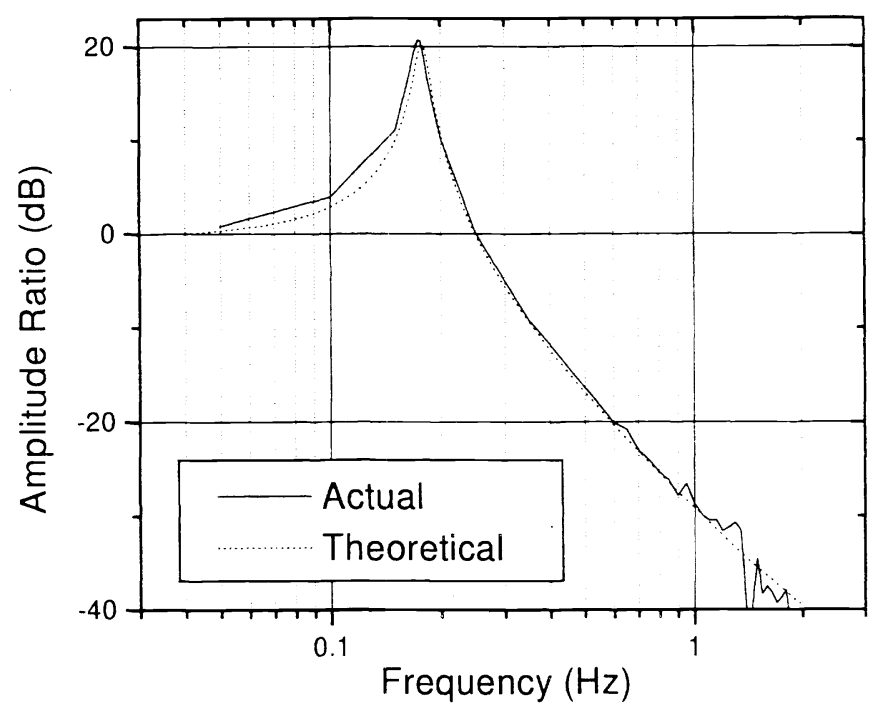

Figure 8. Passive frequency response. Good fit to linear underdamped mass-spring model.

resonance region. The spring constant and damping constant obtained from passive decay match the active driven curve quite accurately. This is the primary validation of our use of a linear model for the system.

\section{1-D FEEDBACK CONTROL SYSTEM}

The actuators have proven to be linear in dynamic response over the available motion range of $\pm 5 \mathrm{~mm}$. This has allowed us to use a linear underdamped mass-spring model for the one-dimensional system. The passive characteristics of following at very low frequency and damping at high frequency will be used to the maximum extent. The control system primarily provides resonance frequency tuning as necessary to adapt to the given environment and additional active damping to remove the resonance overshoot.

\subsection{Control system design criteria}

While the Space Shuttle or the International Space Station will provide a low gravity environment due to the free-fall nature of the orbit, nonetheless there will be residual bias and vibration forces acting throughout the platform. Since there will always be some activity on these platforms, there will be vibrations due to air and liquid handlers, hatches opening and closing, refrigeration pumps, and so on. Each platform will exhibit a different level of vibration, or g-jitter as it is often referred to. In this section, we outline the differences in design strategy required to adapt a one-dimensional laboratory test system to a one-dimensional representation of the Space Shuttle and the KC-135A.

We assume that the g-jitter has a power spectral density exhibiting a relatively uniform envelope across a large frequency range, based on data obtained from a Shuttle flight. There will be a relative displacement limit imposed either by the available space or by the limitations of the measurement devices. This displacement limit will then determine the frequency cutoff below which the experiment box must follow the platform frame or risk impact. Fig. 9 compares the cutoff criteria for vibration levels of the Shuttle versus those of the KC-135A. As can be seen, for the same relative displacement, the cutoff frequency required for the aircraft is much higher than that for the Shuttle. The comparison is necessary for projects needing to go through demonstration/validation on the Reduced Gravity Aircraft prior to deploying on the Shuttle or ISS.

Although the spring constant for the actuator can be adjusted to some extent by varying construction techniques, it is not currently feasible to build an actuator with a specific spring constant value. Also, the inertial mass of the suspended experiment box will vary, case to case. Consequently, we will need to adjust the effective spring constant through electronic feedback to adapt to the specific circumstances. Given a suspended inertial mass of $80 \mathrm{~kg}$, for example, we would need to reduce the spring constant for a lower cutoff for the Shuttle and increase the spring constant for the $\mathrm{KC}-135 \mathrm{~A}$ for a higher cutoff frequency. 


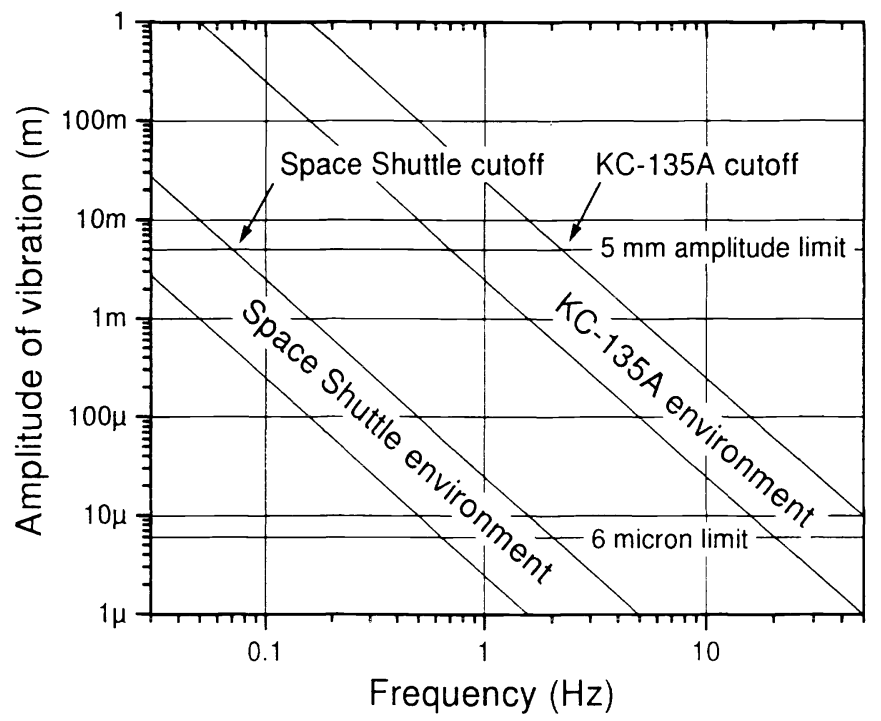

Figure 9. Comparison of vibration environments.

While we will not be able to adjust the effective spring constant over the tremendous range implied by Fig. 9 , we do want to allow as much dynamic range as possible. For demonstration tests on the KC-135A, we will be using a significantly smaller suspended mass than anticipated for the Space Shuttle. The demonstration load will be constrained to approximately $0.5 \mathrm{~kg}$, while the loads on the Shuttle or ISS are expected to be on the order of 80 to $400 \mathrm{~kg}$.

\subsection{Limitations of measurement devices}

Initially, we assumed that accelerometers would be needed as the sensors for control feedback, but the results were highly disappointing. Small, lightweight accelerometers did not have the sensitivity to monitor the low acceleration levels desired and they exhibited a noise level exceeding the signal level desired. Larger, heavier, and more sensitive accelerometers are available, but they are expensive and add significant weight to the experiment. Additionally, positioning the accelerometers to obtain motion data in all six degrees of freedom proved to be a formidable task.

As a replacement to this, we devised a system using only position sensing devices (PSD's). If the only contact to the suspended experiment box is the actuator, then the force on the box is known from the extension of, and the voltage applied to, the actuator and the acceleration can be computed. The addition of viscous friction can be included in the calculation by tracking the rate of change of extension.

For the current design, we found that low-cost, low-power diode lasers, combined with Hamamatsu two-dimensional $12 \mathrm{~mm} \times 12 \mathrm{~mm}$ PSD's give us measurement coverage over the displacement range of the actuators. The sensitivity of the PSD's is on the order of $6 \mu \mathrm{m}$, which is on the order of amplitudes $40 \mathrm{~dB}$ down from the maximum. The pass band of the control system is set to ignore any input with frequencies above the $6 \mu \mathrm{m}$ cutoff. We are using the two-dimensional PSD even in the one-dimensional tests to monitor any out-of-plane motion of the pendulum. In the three-dimensional system, the lasers and PSD's are arranged to form an orthogonal set of axes as will be discussed in Sect. 5 below.

\subsection{Control model}

The actuator and mass are modeled as a linear mass-spring system as shown in Fig. 10. The variables $\mathbf{D}$ and $\mathbf{Y}$ represent the displacement of the platform frame and box, respectively, relative to some (unknown) inertial reference frame. We are interested in the response of the inertial mass of the experiment box as a function of arbitrary motion of the Shuttle frame. The differential equation modeling this system is

$$
\ddot{Y}=-\frac{k}{m}\left(Y-D-y_{e}\right)-\frac{b}{m} \frac{\mathrm{d}}{\mathrm{d} t}(Y-D),
$$




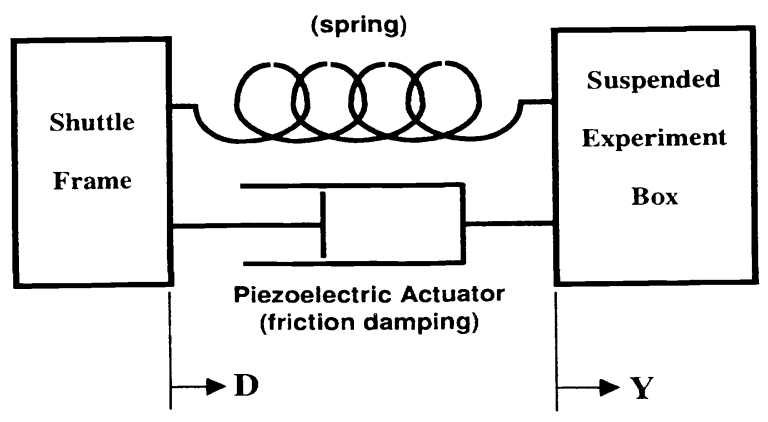

Figure 10. Mass-spring model.

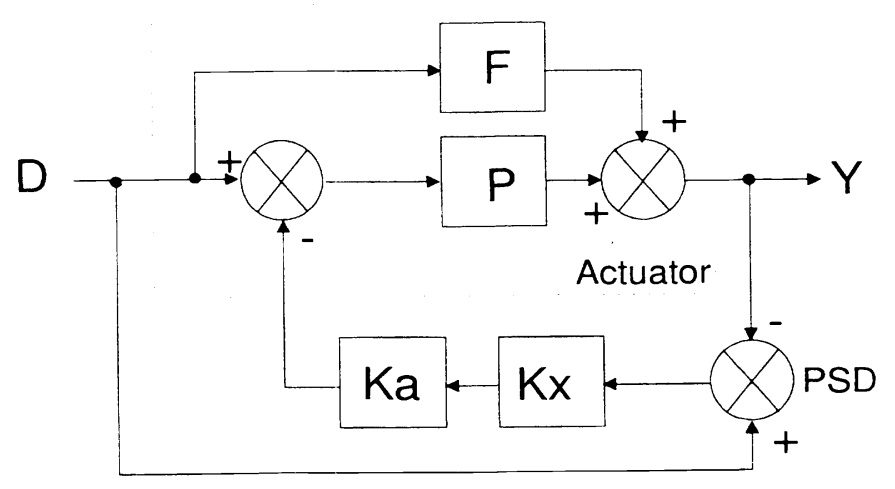

Figure 11. One dimensional system block diagram.

with $y_{e}$ the equilibrium offset of the actuator, which is adjusted by the applied voltage, $m$ is the inertial mass of the suspended box, $k$ is the passive spring constant, and $b$ is the passive damping constant. As the actuators have been shown to be highly linear, we approximate $y_{e}$ as

$$
y_{e}=y_{0}+V d
$$

with $y_{0}$ the equilibrium extension with no applied voltage, $V$ the applied voltage and, $d$ the piezoelectric constant for the complete double bellows actuator stack.

The simplified control model block diagram for the one-dimensional case is shown in Fig. 11. The mass-spring and piezoelectric effects of the actuator are accounted for in the block $\mathbf{P}$. The passive viscous friction coupling between $\mathbf{D}$ and $\mathbf{Y}$ is outside the control system and is represented by the block $\mathbf{F}$. The PSD is capable of measuring the relative displacement $\mathbf{D}-\mathbf{Y}$, and produces a signal of one volt per $\mathrm{mm}$ of relative displacement.

The block Kx represents the principal element of the control system. It consists of a bandpass filter to reject frequencies above and below the range needed for control and a Proportional-plus-Derivative (PD) control module. The proportional response couples to the displacement

$$
\mathbf{x}=\mathbf{D}-\mathbf{Y}+y_{0},
$$

and changes the effective spring constant. The derivative response is based on the time rate of change of the relative displacement $\mathrm{dx} / \mathrm{dt}$, and produces the desired additional (viscous-like) damping.

The output of $\mathbf{K x}$ is scaled in volts per millimeter. The block $\mathbf{K a}$ represents the high voltage amplifier needed to produce a one millimeter extension for a one volt signal produced by the block $\mathbf{K x}$. Specifically, the amplification in $\mathrm{Ka}$ is such that $\mathrm{Ka} \cdot \mathrm{d}=1$. For our prototype actuator stacks, the amplification would be $\sim 200$ from Table 2 . With the reduced electrical stiffness expected from improved manufacturing, this amplification may be reduced by half. 
By making the feedback voltage $V$, created by the block $\mathbf{K} \mathbf{x}$, a linear function of $\mathbf{x}$ and $\mathrm{d} \mathbf{x} / \mathrm{dt}$, with coefficients $K_{x 1}$ and $K_{x 2}$, respectively, we get the form

$$
\ddot{Y}=\frac{k}{m} x+\frac{b}{m} \dot{x}-K_{x 1} \frac{k}{m} x-K_{x 2} \frac{k}{b}\left(\frac{b}{m} \dot{x}\right)
$$

or

$$
\ddot{Y}=\frac{k}{m}\left(1-K_{x 1}\right) x+\frac{b}{m}\left(1-\frac{k}{b} K_{x 2}\right) \dot{x}
$$

With active control, the effective spring constant becomes $k_{e f f}=k\left(1-K_{x 1}\right)$ and the effective damping constant becomes $b_{e f f}=b\left(1-K_{x 2} k / b\right)$. Our goal is to drive the acceleration experienced by the suspended mass to a minimum, consistent with the constraint of not hitting the frame. For example, setting $K_{x 1}=1$ and $K_{x 2}=b / k$ would make the actuator effectively disappear. The box would then not be under any control and would soon hit the frame. For this reason, the function $\mathbf{K x}$ also contains bandpass filters to effect control only around the resonance frequency.

\subsection{Calculation of control variables}

Within the range of voltages allowed by the control system and the actuator, the effective spring constant can be adjusted to produce the desired response. The factors must be calculated for the given mass to be suspended and the specific environment to be experienced. In all conceivable cases, the passive system will be underdamped, so we assume a passive resonance to exist at $\omega_{0}=\sqrt{k / m}$. The proportional term, $K_{x 1}$, is calculated using the passive spring constant $k$ for the actuator, the given inertial mass, $m$ to be controlled, and a desired cutoff frequency, $\omega_{c}=2 \pi f_{c}$, determined from a graph such as Fig. 9. The desired effective spring constant is

$$
k_{e f f}=\omega_{c}^{2} m,
$$

so that

$$
K_{x 1}=1-\frac{m \omega_{c}^{2}}{k} .
$$

The derivative term, $K_{x 2}$, is then determined by calculating the critical damping factor

$$
K_{x 2}=\frac{1}{k}\left(b-2 m \omega_{c}\right)=\frac{b}{k}-\frac{2 \omega_{c}}{\omega_{0}^{2}} .
$$

In practice, we have found that less than critical damping can improve the settling time while not exceeding the acceptable overshoot limits. Additionally, the system response is sensitive to the settings of the bandpass filter limits.

\subsection{Active pendulum tests}

Both analog and digital versions of the control model have been built. Testing primarily focused on the ability to induce additional damping since time derivative circuits and algorithms can be excessively noisy. The results of analog and digital damping are shown in Fig. 12. The additional damping from the digital system is a result of the sharper frequency cutoff possible in the digital bandpass filters.

Without high pass filters the system would become friction dominated and would show only first order roll-off above the cutoff. Without low pass filters, the system would risk saturation attempting to overcome the dc bias. The bandpass filter may need to contain up to fourth order high and low pass elements in the final system to avoid these two problems.

\subsection{Effect of non-zero dc bias accelerations}

On the Shuttle, offset dc bias accelerations on the order of less than $10 \mu \mathrm{g}$ are expected in the space lab module in the cargo bay. For a suspended inertial mass of $80 \mathrm{~kg}$, and four actuators affected by the displacement, we expect less than $0.1 \mathrm{~mm}$ offset from center. Up to $400 \mathrm{~kg}$ would still only produce approximately $1 \mathrm{~mm}$ offset, which would be within tolerance if the effective spring constant were set slightly higher than optimum for no bias. 


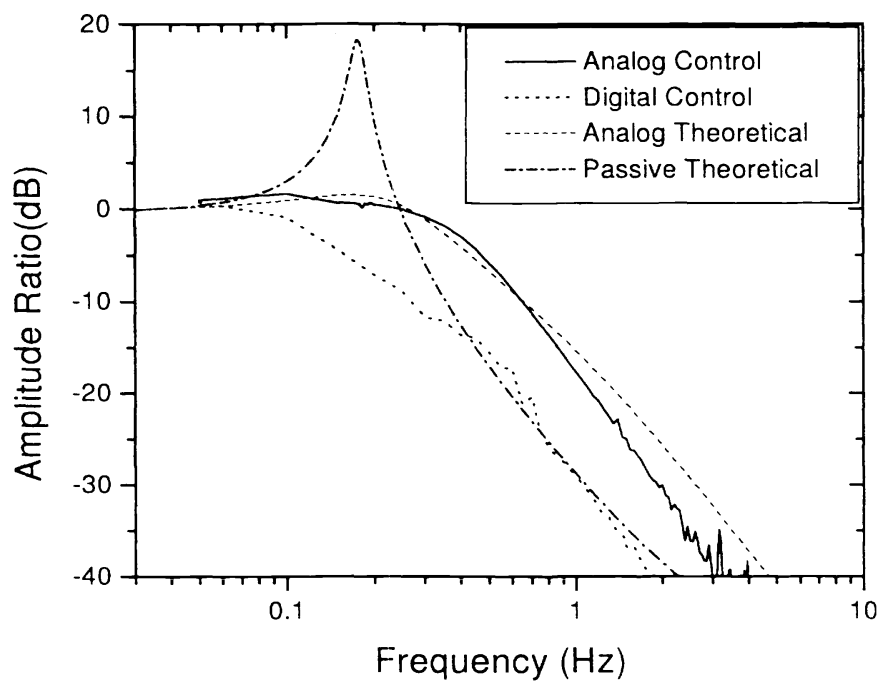

Figure 12. Results with analog and digital damping.

\section{THREE DIMENSIONAL SYSTEM DESIGN}

Throughout development, we have adapted the design to the problems faced with a full six-degree of freedom system. In particular, we have been developing a digital control system which can allow for non-symmetric mass distributions by making use of the proportional adjustment capability in the control model. Knowing the inertia tensor, we can adjust the forces applied by the actuators to create the correct torques under varying spatial configurations due to the motion without making small angle approximations.

\subsection{Solution to 3-d geometry problem}

The need for the small angle approximation was eliminated by solution of the geometry of the relative translation offset and rotation of the box relative to the frame. This calculation has been demonstrated in a PC with up to 500 updates per second without affecting other operations. This rate is well above that required for Space Shuttle applications, but in the range required for demonstrations on the $\mathrm{KC}-135 \mathrm{~A}$.

\subsubsection{Proposed flight test design}

The next flight test configuration calls for 12 actuators arranged in three orthogonal planes of four actuators each around the suspended box. This provides for 24 umbilical signal lines, which is ample for readout of the three 2-dimensional PSD's and a monitoring accelerometer on the box.

\section{CONCLUSIONS}

We have found that the prototype double-bellows actuator stacks behave reasonably in accordance with the analytical and ANSYS simulation results. Although we could potentially achieve improved results with flat structures, the additional manufacturing complexity of adding spacers does not seem justified, given the high degree of linearity observed in the pre-curved structure we are using. We expect the greatest performance improvement from decreasing the thickness of the epoxy layer between PVDF sheets constituting the bimorphs.

Testing in one dimension has verified that our actuators do produce a linear response and can effectively damp out vibrations above a specified cutoff frequency while following average platform motion below the cutoff. The use of a contact system such as this should be compared with non-contact proposals such as in Ref. 11, which discusses use of magnetic actuators. Magnetic systems provide better passive damping in general but also tend to be heavier than the small polymer devices described here. Also, non-contact systems force a separate solution to the umbilical problem. 
The simplified control seems to couple to the physics of the motion quite adequately, at least in one dimension, but modeling indicates that the use of position control, plus derived velocity control, should be readily adapted to the three-dimensional case as well.

\section{ACKNOWLEDGMENTS}

This work has been supported by NASA EPSCoR Grant NCC5-240 and by the Montana Space Grant Consortium. We would also like to acknowledge the assistance of D. G. Blair in the adaptation of the folded pendulum for our purposes.

\section{REFERENCES}

1. V. H. Schmidt, J. Conant, G. Bohannan, J. Eckberg, S. Halko, J. Hallenberg, C. Nelson, N. Peterson, C. Smith, C. Thrasher, and B. Tikalsky, "Piezoelectric polymer actuators for vibration suppression," Proc. of SPIE 3669, pp. 162-170, 1999.

2. G. Bohannan, V. H. Schmidt, D. Brandt, and M. Mooibroek, "Piezoelectric polymer actuators for active vibration isolation in space applications," Ferroelectrics 224, pp. 211-217, 1999.

3. D. S. Stampleman, "Microgravity isolation mount based on piezoelectric film," Proc. SPIE 1898, pp. 716-731, 1993.

4. B. A. Auld, Acoustic Fields and Waves in Solids, vol. 1, p. 271. Krieger Publishing Co., Malabar, FL, 2 ed., 1990.

5. H. Wang, M. Zhang, and L. E. Cross, "Piezoelectric, dielectric and elastic properties of poly(vinylidene fluoride/trifluoroethylene)," J. Appl. Phys. 74, pp. 3394-3398, 1993.

6. A. E. Childs, "Modeling and analysis of thin-film, piezoelectric actuators," Master's thesis, Montana State University, 2000.

7. R. M. Jones, Mechanics of Composite Materials, p. 40. McGraw-Hill Book Co., New York, 1975.

8. D. I. Jones, "A control system for a microgravity isolation mount," Proc. 1990 Amer. Control Conf. , pp. 2601$1606,1990$.

9. D. G. Blair, J. Liu, and E. F. Moghaddam, "Performance of an ultra-low frequency folded pendulum," Phys. Lett. A 193, pp. 223-226, 1994.

10. J. Liu, J. Winterflood, and D. G. Blair, "Transfer function of an ultra low frequency vibration isolation system," Rev. Sci. Instrum. 66, pp. 3216-3218, 1995.

11. D. I. Jones, "A control system for a microgravity isolation mount," IEEE Trans. Control Sys. Techn. 4, pp. 313$325,1996$. 